\title{
The early build-up of dust in galaxies: A study of damped Ly $\alpha$ systems
}

\begin{abstract}
G. Vladilo^
Osservatorio Astronomico di Trieste - Istituto Nazionale di Astrofisica, via G.B. Tiepolo 11, 34131 Trieste, Italy

Received 18 December 2003 / Accepted 10 March 2004

Abstract. We present a study of the early build-up of dust in high redshift galaxies. The study is based on the analysis of 38 Damped Ly $\alpha$ systems (DLAs) for which we derive the fraction of iron atoms in dust form, $f_{\mathrm{Fe}}$. The sample is representative of metal-poor galaxies in the redshift range $0.6 \leq z \leq 3.4$ selected on the basis of their absorption H I column density $\left(N\left(\mathrm{H}_{\mathrm{I}}\right) \geq\right.$ $2 \times 10^{20}$ atoms $\mathrm{cm}^{-2}$ ). We find that the dust fraction increases with metallicity, from $f_{\mathrm{Fe}} \simeq 0$ at $[\mathrm{Fe} / \mathrm{H}] \sim-2$ dex, up to $f_{\mathrm{Fe}} \simeq 0.9$ at solar metallicity; the increase is fast below $[\mathrm{Fe} / \mathrm{H}] \simeq-1$ dex and mild at higher metallicities. We also find some evidence for an increase of $f_{\mathrm{Fe}}$ with cosmic time; a large fraction of the systems younger than $\approx 3 \mathrm{Gyr}$ has $f_{\mathrm{Fe}} \lesssim 0.5$. These results indicate that the dust-to-metal ratio increases in the course of chemical evolution, at variance with the hypothesis of an approximately constant dust-to-metal ratio, commonly adopted in models of galactic evolution. This hypothesis is consistent with local and high-redshift data only when the metallicity is relatively high $([\mathrm{Fe} / \mathrm{H}] \gtrsim-1 \mathrm{dex})$. The results of this work suggest that the main mechanisms of dust formation may be rather sensitive to the level of metallicity attained by a galaxy in the course of its chemical evolution. A metallicity-dependent dust production by SNe II seems to be the most promising mechanism to explain the rise of $f_{\mathrm{Fe}}$ at $[\mathrm{Fe} / \mathrm{H}] \lesssim-1 \mathrm{dex}$.
\end{abstract}

Key words. ISM: dust, extinction - galaxies: ISM - galaxies: evolution - galaxies: high-redshift galaxies: quasars: absorption lines

\section{Introduction}

Interstellar dust plays a key role in a variety of astrophysical processes relevant to galactic evolution and affects, with its presence, many measurements of basic physical properties of stars and galaxies. For these reasons, understanding the process of dust formation and evolution on a galactic scale is of great interest in several areas of astrophysical research. In the course of galactic evolution the abundance of dust is expected to track that of the metals synthesized and ejected from the stars. Therefore, the dust-to-gas ratio, $\mathcal{D}$, is expected to correlate with the metallicity, $\mathcal{Z}$ or, in other words, the fraction of heavy elements in dust form, $f_{\mathrm{d}} \approx \mathcal{D} / \mathcal{Z}$, is expected to stay approximately constant (e.g., Franco \& Cox 1986). In the local universe the existence of a $\mathcal{Z}-\mathcal{D}$ correlation is supported by empirical evidence. For instance, Issa et al. (1990) found a good correlation for 6 spirals of the Local Group and the Magellanic Clouds, estimating $\mathcal{D}$ from the visual extinction per hydrogen atom. Schmidt \& Boller (1993) found a similar correlation for 23 local dwarf galaxies estimating $\mathcal{D}$ from IRAS dust mass and H I integrated-emission mass. From a similar analysis, Lisenfeld \& Ferrara (1998) found a good correlation for 28 dwarf irregular galaxies, but not for 16 blue compact dwarfs; they attribute these differences to variations of the star-formation and mass-outflow rates between these different types of galaxies.

\footnotetext{
* e-mail: vladilo@ts.astro.it
}

The empirical $\mathcal{Z}-\mathcal{D}$ correlation found in the local universe is, to date, the main observational input to models of galactic evolution that incorporate the dust component. A selfconsistent treatment of the metal and dust content in the models requires taking into account the processes of grain formation, accretion and destruction (e.g., Dwek \& Scalo 1980), increasing in such a way the number of the parameters with few, if any, observational constraints. The detailed treatment of all these processes can be performed using numerical models. Following this approach, and requiring that accretion and destruction time-scales evolve in a similar fashion, Dwek (1998) found that the interstellar $\mathcal{D} / \mathcal{Z}$ mass ratio is approximately constant in the Milky Way. More elementary, analytical models have been recently proposed to reproduce the dust cycle in galaxies (e.g., Hirashita 1999; Edmunds 2001; Inoue 2003). An approximately constant $\mathcal{D} / \mathcal{Z}$ mass ratio is generally predicted by these studies, with the exception of Inoue (2003), who proposes models with $\mathcal{D} / \mathcal{Z}$ increasing in the course of evolution. The observed linear $\mathcal{Z}-\mathcal{D}$ relation in the local universe is interpreted by Inoue as a sequence of constant galactic age. Measuring the dust-to-metal ratios in galaxies caught in their early stages of evolution is fundamental to probe the validity of these models. This can be done by studying the dust content of high-redshift galaxies, a field of research that is receiving special attention given its potential impact on our understanding of the early universe (e.g., Silva et al. 1998; Hirashita \& Ferrara 2002; Morgan \& Edmunds 2003). 
Quasar absorption-line systems can be used to investigate the relation between dust and metallicity at high redshift based on observations. This is true for the Damped Ly $\alpha$ (DLA) systems, the class of absorbers with the highest H I column density $\left(N(\mathrm{H} \mathrm{I})>10^{20.3}\right.$ atoms $\left.\mathrm{cm}^{-2}\right)$, which are believed to originate in galaxies located along the quasar line of sight (Wolfe et al. 1986). Measuring the metallicity and the dust abundance of DLA systems makes it possible to probe the $\mathcal{D}-\mathcal{Z}$ relation in primeval galaxies. The metallicity is determined from column-density measurements, which can be quite accurate (e.g., Lu et al. 1996; Prochaska \& Wolfe 1999; Molaro et al. 2000; Ledoux et al. 2003). Element abundances do not suffer from severe ionization corrections in DLA systems (e.g., Vladilo et al. 2001), and the metallicity level can be estimated well, at least for elements not affected by dust depletion (Pettini et al. 1999). Measuring the dust abundance, however, is less straightforward.

In principle, the amount of dust in DLA systems can be estimated from the reddening of the background quasar. This technique, however, cannot be applied to individual systems given the uncertain, variable continuum of the quasar. As a consequence, quasar extinction has only been estimated statistically, by comparing samples with and without foreground DLAs (Pei et al. 1991). The dust-to-gas ratio in DLA systems estimated in this way is between $5 \%$ and $20 \%$ of the Galactic value, in broad agreement with the low level of metallicity of DLA systems. However, this type of analysis does not give information on the evolution of the dust abundance with the metallicity. Also the dust emission properties cannot be employed to derive the dust abundance in DLA systems. Attempts to identify the emission of intervening galaxies in the quasar field have been successful only for a dozen absorbers at $z \lesssim 1$ (Le Brun et al. 1997; Turnshek et al. 2001; see also Kanekar \& Chengalur 2003), but not in the redshift interval where DLAs are most commonly identified and investigated $(z \gtrsim 2)$.

So far, the only method to estimate the dust abundance of individual DLAs relies on the comparison of the elemental abundances of refractory and non-refractory elements. Since the pioneer investigation on $\mathrm{Cr}$ and $\mathrm{Zn}$ by Pettini et al. (1994), the evidence for differential depletion of refractory elements relative to the volatile element $\mathrm{Zn}$ has accumulated over the years and is now well established (Hou et al. 2001; Prochaska $\&$ Wolfe 2002). An additional argument supporting the connection between depletion and dust is the correlation between depletion and fraction of molecular hydrogen (Levshakov et al. 2000; Ledoux et al. 2003).

The existence of a correlation between differential depletion and metallicity, reported by Ledoux et al. (2003), suggests that the dust content of DLA systems evolves in the course of chemical evolution. However, to study the evolutionary properties of the dust we need to convert the differential depletions into dust-to-gas or dust-to-metal ratios. In a previous work we presented a method for estimating these ratios in DLA systems, taking also into account the depletion of $\mathrm{Zn}$ self-consistently (Vladilo 1998, Paper I). In that work we derived a $\mathcal{Z}-\mathcal{D}$ correlation between the metallicity corrected for dust effects and the dust-to-gas ratio. These two quantities, however, are not independent when the observed metallicity is very low. A much safer approach consists of studying the evolution of the dust-tometal ratio, rather than that of the dust-to-gas ratio. The former is derived from the column densities of two metals and is less affected by error propagation than the latter, which requires, in addition, the use of the H I column density.

A systematic study of the evolution of the dust-to-metal ratio in DLA systems is still lacking. This type of study requires converting the measured differential depletions into the fraction of metals in dust form, $f_{\mathrm{d}}$, taking into account (i) variations of the dust composition that may occur in different types of galactic environments (e.g. galaxies with different metallicities) and (ii) nucleosynthetic effects that may affect the volatile/refratory abundance ratio (e.g. evolution of the $\mathrm{Zn} / \mathrm{Fe}$ ratio). In a recent work we presented a methodology aimed at tackling these problems (Vladilo 2002a, Paper II). This methodology was successfully applied to recover (i) the (stellar) abundance pattern of the Small Magellanic Cloud starting from interstellar measurements affected by dust depletion; (ii) the $\alpha / \mathrm{Fe}$ ratio in DLA systems corrected for dust effects (Vladilo 2002b, Paper III).

In the present paper we apply this new method to derive the fraction of iron in dust form, $f_{\mathrm{Fe}}$, for the sample of DLA systems with measurements of the $\mathrm{Zn} / \mathrm{Fe}$ ratio. This sample now includes 38 absorbers, more than twice the sample of Paper I. We use the results of the most recent studies of Zn abundance in metal-poor stars to estimate the intrinsic $\mathrm{Zn} / \mathrm{Fe}$ ratio in DLA systems which, at variance with Paper I, is now a free parameter of the method. Finally, for the first time in this type of investigation, the robustness of the results is tested against possible bias introduced by the observations or by the method itself. The paper is organized as follows. In the next section we present the method of analysis and the sample. In Sect. 3 we present the results and test their robustness. In Sect. 4 we discuss the implications of the results in terms of formation and evolution of dust in primeval galaxies. The conclusions are summarized in Sect. 5.

\section{Analysis of the observational data}

\subsection{The method}

To study the evolutionary properties of dust in DLA systems we measure the fraction of metals in dust form in each system. We call dust fraction of an element $\mathrm{X}$ the number ratio

$f_{\mathrm{X}}=N_{\text {dust }}(\mathrm{X}) / N_{\text {tot }}(\mathrm{X})$,

where $N_{\text {dust }}(\mathrm{X})$ and $N_{\text {tot }}(\mathrm{X})$ are the column densities of $\mathrm{X}$ (atoms $\mathrm{cm}^{-2}$ ) in dust form and in the medium (gas plus dust), respectively. The dust fraction of a metal indicates how efficient the ISM of a galaxy is in incorporating part of its heavy elements into dust grains and represents, in practice, a dustto-metal ratio by number. Here we adopt the dust fraction of iron, $f_{\mathrm{Fe}}$, as an estimator of the dust-to-metal ratio in DLA systems. Iron is a natural choice since it is easy to measure in DLAs and is a reference element used in different types of abundance studies. Most important, iron is a refractory element which traces the dust even in the harshest interstellar conditions, where dust grains tend to be destroyed. This is demonstrated by the fact that in the warm halo gas of the Milky Way, 
where the level of depletion is known to be generally low, the dust fraction of iron is still quite high, $f_{\mathrm{Fe}, w h} \simeq 0.77$ (Savage \& Sembach 1996).

\subsubsection{Derivation of the dust fraction of iron}

The dust fraction of iron can be derived by comparing the observed column density of iron with that of a volatile element $\mathrm{X}$ if we can make an educated guess of the intrinsic abundance ratio X/Fe in the DLA system. The method works best when the differential dust depletion between $\mathrm{Fe}$ and $\mathrm{X}$ is high. The most natural choice for $\mathrm{X}$ is $\mathrm{Zn}$, a volatile element that tracks Fe well in the course of nucleosynthetic evolution (see Sect. 2.2.3). If we call $[\mathrm{Zn} / \mathrm{Fe}]_{\mathrm{g}}$ the educated guess of the intrinsic ratio in DLAs, we can use the equation

$\varrho-\frac{f_{\mathrm{Zn}, i} 10^{\left[\frac{\mathrm{Zn}}{\mathrm{Fe}}\right]_{\mathrm{g}} \varepsilon_{\mathrm{Zn}}}}{f_{\mathrm{Fe}, i} 10^{\left[\frac{\mathrm{Zn}}{\mathrm{Fe}}\right]}} \varrho^{\left(1+\eta_{\mathrm{Zn}}\right)}+\frac{10^{\left[\frac{\mathrm{Zn}}{\mathrm{Fe}}\right]_{\mathrm{g}}}-10^{\left[\frac{\mathrm{Zn}}{\mathrm{Fe}}\right]}}{f_{\mathrm{Fe}, i} 10^{\left[\frac{\mathrm{Zn}}{\mathrm{Fe}}\right]}}=0$,

derived in Paper III to obtain the normalized dust-to-metal ratio $\varrho=f_{\mathrm{Fe}} / f_{\mathrm{Fe}, i}$ from the ratio $[\mathrm{Zn} / \mathrm{Fe}]$ measured in the $\mathrm{DLA}^{1}$. In the above equation the fractions in dust $f_{\mathrm{Fe}, i}$ and $f_{\mathrm{Zn}, i}$ are measured in one particular phase $i$ of the Galactic ISM (e.g. cold gas or warm gas in the disk or in the halo). Without loss of generality, we adopt here the warm gas in the disk as a reference value: we stress that, at variance with Paper I, the present method allows for variation of the dust composition among different interstellar phases (see Paper II). These variations are taken into account by the parameter $\eta_{\mathrm{Zn}}$, which describes how the $\mathrm{Zn} / \mathrm{Fe}$ ratio in the dust varies as a function of $\varrho$. The parameter $\eta_{\mathrm{Zn}}$ is determined from studies of the Galactic depletion patterns (Paper II). The method also allows the dust composition to vary as a function of the composition of the medium. This dependence is accounted for by the parameter $\varepsilon_{\mathrm{Zn}}$, discussed below. Finally, we stress that Eq. (2) takes into account in a self-consistent way the fact that $\mathrm{Zn}$ also is partly depleted, while dust depletion studies in the literature ignore this effect.

Equation (2) is a transcendent equation in the unknown $\varrho$ that we solve by iterations. The equation has a unique solution inside the interval $0<\varrho \leq \varrho_{\lim }$, where $\varrho_{\lim }$ is the maximum possible value of $\varrho$, corresponding to a dust fraction of iron of $100 \%$. Solutions outside this interval are not physical.

\subsubsection{Derivation of the metallicity}

Once the dust fraction of iron is known, it is straightforward to derive the total column density of iron (gas plus dust) and from this the iron abundance corrected for dust effects, $[\mathrm{Fe} / \mathrm{H}]_{\mathrm{c}}$, used here as the indicator of metallicity. In most cases the dustcorrected metallicity $[\mathrm{Fe} / \mathrm{H}]_{\mathrm{c}}$ is very similar to the zinc abundance $[\mathrm{Zn} / \mathrm{H}]$, which is commonly adopted in the literature as the indicator of metallicity in DLA systems, with a typical difference $[\mathrm{Fe} / \mathrm{H}]_{\mathrm{c}}-[\mathrm{Zn} / \mathrm{H}] \lesssim+0.1$ dex.

\footnotetext{
${ }^{1}[\mathrm{Zn} / \mathrm{Fe}] \equiv \log \{N(\mathrm{Zn}) / N(\mathrm{Fe})\}-\log (\mathrm{Zn} / \mathrm{Fe})_{\odot}$, where $N(\mathrm{Zn})$ and $N(\mathrm{Fe})$ are the column densities derived from spectroscopic measurements. The reference solar abundance by number, $\log (\mathrm{Zn} / \mathrm{Fe})_{\odot}=$ -2.86 , is taken from meteoritic measurements (Anders \& Grevesse 1989).
}

\subsection{The parameters}

\subsubsection{The dust fraction of zinc in the Galactic ISM}

Local interstellar studies indicate that a fraction of zinc is incorporated in dust form even in lines of sight with moderate level of depletion. In Paper II we derived the dust fraction of zinc in the Galactic warm disk gas, $f_{\mathrm{Zn}, w d}=0.59$, using the same lines of sight with accurate HST measurements adopted by Savage \& Sembach (1996) in their study of depletion patterns. Unfortunately, that sample of lines of sight included only a few zinc determinations. For this reason, we now derive $f_{\mathrm{Zn}, w d}$ also using the largest compilation of HST measurements of zinc and chromium (Roth \& Blades 1995). As a result, we obtain $f_{\mathrm{Zn}, w d}=0.30$ using the same scaling law of interstellar depletion adopted in Paper II and imposing that the dust-corrected abundance of $\mathrm{Cr}$ in the local ISM is solar. We consider that the range $0.30 \leq f_{\mathrm{Zn}, w d} \leq 0.59$ is fairly representative of the uncertainty of this parameter.

\subsubsection{The parameter $\varepsilon_{\mathrm{Zn}}$}

The parameter $\varepsilon_{\mathrm{Zn}}$ describes how the $\mathrm{Zn} / \mathrm{Fe}$ ratio in the dust composition varies as a function of the same ratio in the composition of the medium. By definition, $\varepsilon_{\mathrm{Zn}}=1$ if a percent variation of the $\mathrm{Zn} / \mathrm{Fe}$ ratio in the medium yields the same percent variation in the dust (Paper II). If, on the other hand, the $\mathrm{Zn} / \mathrm{Fe}$ ratio in the dust is totally independent of variations of the ratio in the medium, then $\varepsilon_{\mathrm{Zn}}=0$. In principle, $\varepsilon_{\mathrm{Zn}}$ can be derived studying depletion patterns in galaxies with a known chemical composition. A preliminary study of SMC data is consistent with $\varepsilon_{\mathrm{Zn}} \simeq 1$. Here we consider both cases $\varepsilon_{\mathrm{Zn}}=1$ and $\varepsilon_{\mathrm{Zn}}=0$ as extreme possibilities to solve Eq. (2) starting from reference Galactic ISM values. Given the form of Eq. (2) in which $\varepsilon_{\mathrm{Zn}}$ multiplies $[\mathrm{Zn} / \mathrm{Fe}]_{\mathrm{g}}$, the choice of $\varepsilon_{\mathrm{Zn}}$ is not critical if the intrinsic $\mathrm{Zn} / \mathrm{Fe}$ ratio is approximately solar. Luckily, the $\mathrm{Zn} / \mathrm{Fe}$ is indeed close to solar and this explains why the results presented below are largely independent of the choice of $\varepsilon_{\mathrm{Zn}}$.

\subsubsection{The $\mathrm{Zn} / \mathrm{Fe}$ ratio in DLA systems}

To make an educated guess of the $\mathrm{Zn} / \mathrm{Fe}$ ratio in DLA systems we must rely on abundance studies of metal-poor stars. The early studies of Galactic stars yielded a $\mathrm{Zn} / \mathrm{Fe}$ ratio close to solar (Sneden et al. 1991), but some recent work indicates that the $\mathrm{Zn} / \mathrm{Fe}$ ratio can be overabundant at very low metallicity (Primas et al. 2000; Prochaska \& Wolfe 2002). The latter results have been used to support the notion that the $\mathrm{Zn} / \mathrm{Fe}$ ratio may decrease in the course of chemical evolution owing to a different nucleosynthetic origin of $\mathrm{Zn}$ and $\mathrm{Fe}$ (see e.g., Umeda \& Nomoto 2002).

A large number of $\mathrm{Zn}$ abundance measurements have been recently published by three different groups, all indicating that the $\mathrm{Zn} / \mathrm{Fe}$ ratio is very close to solar, or slightly enhanced, in the range of metallicities typical of DLA systems (Mishenina et al. 2002; Gratton et al. 2003; Nissen et al. 2004). The 87 measurements performed by Mishenina et al., derived from spectra collected at the 1.93-m telescope of Haute Provence, yield 
a mean value $\langle[\mathrm{Zn} / \mathrm{Fe}]\rangle=+0.08 \pm 0.13 \mathrm{dex}(1 \sigma)$ in the interval $-3 \leq[\mathrm{Fe} / \mathrm{H}] \leq-0.2$. The 48 measurements obtained from UVES/VLT spectra by Gratton et al. yield $\langle[\mathrm{Zn} / \mathrm{Fe}]\rangle=$ $+0.13 \pm 0.13$ dex in the interval $-2.4 \leq[\mathrm{Fe} / \mathrm{H}] \leq-0.3$. The 29 measurements by Nissen et al., also obtained with UVES/VLT data, yield $\langle[\mathrm{Zn} / \mathrm{Fe}]\rangle=+0.04 \pm 0.08 \mathrm{dex}$ in the interval $-2.4 \leq[\mathrm{Fe} / \mathrm{H}] \leq-0.7$. The scales of the zinc abundances in these measurements might be in error up to $0.1 \mathrm{dex}$ owing to differences between the photospheric and meteoritic solar abundance. For instance, Gratton et al. used a reference solar ratio $\log (\mathrm{Zn} / \mathrm{Fe})_{\odot}=-2.95$ derived from a consistent analysis of the solar spectrum. Using instead the solar meteoritic value $\log (\mathrm{Zn} / \mathrm{Fe})_{\odot}=-2.86$ (Anders \& Grevesse 1989), one would derive $\langle[\mathrm{Zn} / \mathrm{Fe}]\rangle=+0.04 \pm 0.13$ dex from Gratton et al. data. Also a systematic difference in the scale of effective temperature could easily account for the mean offset relative to the solar value.

The $[\mathrm{Zn} / \mathrm{Fe}]$ data do not show a trend with metallicity, with the possible exception of a very weak decrease with $[\mathrm{Fe} / \mathrm{H}]$ in the Nissen et al. sample and in the sub-sample of halo stars of Mishenina et al., neither statistically significant. Therefore a constant value of $[\mathrm{Zn} / \mathrm{Fe}]_{\mathrm{g}}$ is a reasonable choice in the metallicity interval typical of DLA systems. We considered two possible values, namely $[\mathrm{Zn} / \mathrm{Fe}]_{\mathrm{g}}=0$, representative of the solar value, and $[\mathrm{Zn} / \mathrm{Fe}]_{\mathrm{g}}=+0.1$, representative of a modest enhancement. The potential effects of a hypothetic decrease of $\mathrm{Zn} / \mathrm{Fe}$ with $\mathrm{Fe} / \mathrm{H}$ are considered in the discussion.

\subsection{The sample}

The data used in the present investigation were selected by searching for DLA systems with published measurements of Fe II and Zn II column densities. All systems with $N(\mathrm{H}$ I) in excess of the canonical threshold $N(\mathrm{HI})=10^{20.3}$ atoms $\mathrm{cm}^{-2}$, or consistent with the threshold at $\simeq 1 \sigma$ level, were included in the sample. Upper limits were excluded. The resulting sample, listed in Table 1, includes 38 systems in the redshift interval $0.6 \leq z \leq 3.4$. The references to the original measurements are given in the table. Most of the measurements were obtained using high resolution spectrographs fed by $8-10 \mathrm{~m}$ class telescopes such as the Keck and the VLT. Care has been taken in checking that all the column densities are derived with an updated and consistent set of oscillator strengths (Bergeson \& Lawler 1993; Welty et al. 1999).

\section{Results}

In the last 4 columns of Table 1 we list the fraction in dust of iron in DLA systems $f_{\mathrm{Fe}}=\varrho f_{\mathrm{Fe}, w d}$ obtained from Eq. (2) for different choices of the input parameters $f_{\mathrm{Zn}, w d}, \varepsilon_{\mathrm{Zn}}$ and $[\mathrm{Zn} / \mathrm{Fe}]_{\mathrm{g}}$, specified in the notes to the table. The quoted errors have been derived by propagating the Fe II and Zn II columndensity errors. The results obtained for a solar $[\mathrm{Zn} / \mathrm{Fe}]_{\mathrm{g}}$ are labeled $S$; those for an enhanced $[\mathrm{Zn} / \mathrm{Fe}]_{\mathrm{g}}(=+0.1)$ are labeled $\mathrm{E}$. For the case $\mathrm{S}$ we list the results obtained using $f_{\mathrm{Zn}, w d}=0.59$ and $f_{\mathrm{Zn}, w d}=0.30$, labeled $\mathrm{Sa}$ and $\mathrm{Sb}$, respectively. The differences are in most cases well below the quoted errors. The results $S$ do not depend on the adopted value of $\varepsilon_{Z n}$ (see Eq. (2)).
The results $\mathrm{E}$ do depend on this parameter and are labeled E0 and $\mathrm{E} 1$ to indicate the cases $\varepsilon_{\mathrm{Zn}}=0$ and $=1$, respectively. Comparing the columns E0 and E1 of Table 1 one can see that the dependence on $\varepsilon_{\mathrm{Zn}}$ is indeed weak, as anticipated above.

\subsection{Frequency distribution of depletions}

In Fig. 1 we show the frequency distribution of iron depletions $^{2}$,

$\delta_{\mathrm{Fe}}=\log \left(1-f_{\mathrm{Fe}}\right)$.

For comparison with Galactic interstellar studies we also plot the depletions representative of the Galactic cold disk gas (dashed-dotted line), the warm disk gas (dashed line) and the warm halo gas (dotted line), taken from Savage \& Sembach (1996).

The frequency distributions are shown for different choices of input parameters (cases $\mathrm{Sa}, \mathrm{Sb}$ and $\mathrm{E} 1$ ). The main results common to all cases can be summarized as follows. The relative number of systems decreases with increasing depletion $\left|\delta_{\mathrm{Fe}}\right|$. A large fraction of systems have depletions lower than the lowest Galactic depletions (those of the warm halo gas; dotted line). The remaining DLAs have depletions similar to those of the warm halo or disk gas; only for these cases is it correct to conclude that "depletions in DLA systems are typical of warm Galactic gas", a claim often quoted in the literature. Cold disk depletions are not found in the sample. These conclusions are also valid for the case E0, not shown in the figure.

\subsection{Dust fraction versus metallicity}

We next consider the relation between dust fraction, $f_{\mathrm{Fe}}$, and metallicity, which is shown in Fig. 2 for three different choices of input parameters (cases $\mathrm{Sa}, \mathrm{Sb}$ and E1). A very similar relation is found for the case E0, not shown in the figure. The main result, common to all cases considered, is that the dust fraction increases with metallicity, with a flattening at $[\mathrm{Fe} / \mathrm{H}]_{\mathrm{c}} \gtrsim-1 \mathrm{dex}$ and a significant scatter. The data follow an empirical law of the type

$f_{\mathrm{Fe}} \approx f_{\mathrm{Fe}, \circ}-10^{\alpha\left([\mathrm{Fe} / \mathrm{H}]-[\mathrm{Fe} / \mathrm{H}]_{\circ}\right)}$

with $f_{\mathrm{Fe}, \circ} \simeq 0.9, \alpha \simeq-1.2$, and $[\mathrm{Fe} / \mathrm{H}]_{\circ} \simeq-1.7$ (solid curve in the figures). The upper (lower) envelope of the data, shown in Fig. 3b, can be represented with the same law, with parameters $f_{\mathrm{Fe}, \circ} \simeq 0.99(0.67), \alpha \simeq-1(-4)$, and $[\mathrm{Fe} / \mathrm{H}]_{\circ} \simeq-1.7(-2.15)$.

The comparison with local ISM depletions reveals that the systems with depletions lower than in the Galactic halo gas are mostly found at $[\mathrm{Fe} / \mathrm{H}]_{\mathrm{c}} \lesssim-1 \mathrm{dex}$.

We stress that the increase of $f_{\mathrm{Fe}}$ with metallicity is confirmed even assuming that $[\mathrm{Zn} / \mathrm{Fe}]_{g}$ evolves from $\approx+0.2 /+$ 0.3 dex at $[\mathrm{Fe} / \mathrm{H}] \lesssim-2$ dex, down to $\simeq 0$ at solar metallicity, as claimed by some authors (see Sect. 2.2.3). In this case we would obtain even less dust than in Fig. 2 c at $[\mathrm{Fe} / \mathrm{H}] \lessgtr-2$ dex, but the same amount of dust as in Fig. $2 \mathrm{a}$ at solar metallicity.

\footnotetext{
2 It is easy to show that Eq. (3) is equivalent to the classical definition $\delta_{\mathrm{Fe}}=\log \left[N(\mathrm{Fe})_{\mathrm{obs}} / N(\mathrm{H})_{\mathrm{obs}}\right]-\log (\mathrm{Fe} / \mathrm{H})_{\odot}$, when the intrinsic abundance of the medium is solar, as in the Galactic ISM.
} 
Table 1. Fraction of iron in dust, $f_{\mathrm{Fe}}$, in damped Ly $\alpha$ systems estimated for different sets of input parameters ${ }^{a}$.

\begin{tabular}{|c|c|c|c|c|c|c|c|c|c|}
\hline Identifier & $z$ & $\log N\left(\mathrm{H}_{\mathrm{I}}\right)^{b}$ & $\log N(\mathrm{Fe} \mathrm{II})^{b}$ & $\log N(\mathrm{Zn} \text { II })^{b}$ & References & $f_{\mathrm{Fe}}(\mathrm{Sa})^{c}$ & $f_{\mathrm{Fe}}(\mathrm{Sb})^{d}$ & $f_{\mathrm{Fe}}(\mathrm{E} 0)^{e}$ & $f_{\mathrm{Fe}}(\mathrm{E} 1)^{f}$ \\
\hline $0000-263$ & 3.3901 & $21.41 \pm 0.08$ & $14.77 \pm 0.03$ & $12.01 \pm 0.05$ & 13 & $0.215_{-0.113}^{+0.100}$ & $0.215_{-0.113}^{+0.099}$ & $0.011_{-0.011}^{+0.124}$ & $0.011_{-0.011}^{+0.124}$ \\
\hline 0013-004 & 1.9731 & $20.70 \pm 0.05$ & $14.37 \pm 0.01$ & $12.63 \pm 0.06$ & 6,16 & $0.979_{-0.005}^{+0.004}$ & $0.948_{-0.009}^{+0.008}$ & $0.953_{-0.010}^{+0.008}$ & $0.971_{-0.007}^{+0.006}$ \\
\hline $0058+019$ & 0.6125 & $20.08 \pm 0.15$ & $15.24 \pm 0.04$ & $12.81 \pm 0.13$ & 18 & $0.669_{-0.165}^{+0.132}$ & $0.646_{-0.149}^{+0.111}$ & $0.547_{-0.186}^{+0.147}$ & $0.552_{-0.190}^{+0.156}$ \\
\hline 0058-2914 & 2.6711 & $21.20 \pm 0.15$ & $14.96 \pm 0.14$ & $13.04 \pm 0.15$ & 2 & $0.961_{-0.054}^{+0.020}$ & $0.916_{-0.066}^{+0.036}$ & $0.915_{-0.090}^{+0.041}$ & $0.941_{-0.089}^{+0.032}$ \\
\hline $0100+130$ & 2.3090 & $21.40 \pm 0.05$ & $14.99 \pm 0.04$ & $12.45 \pm 0.02$ & 24 & $0.534_{-0.060}^{+0.056}$ & $0.525_{-0.056}^{+0.052}$ & $0.396_{-0.070}^{+0.064}$ & $0.397_{-0.070}^{+0.065}$ \\
\hline $0149+33$ & 2.1400 & $20.50 \pm 0.10$ & $14.23 \pm 0.02$ & $11.50 \pm 0.10$ & 24 & $0.252_{-0.204}^{+0.166}$ & $0.252_{-0.204}^{+0.163}$ & $0.058_{-0.058}^{+0.202}$ & $0.058_{-0.058}^{+0.202}$ \\
\hline $0201+365$ & 2.4620 & $20.38 \pm 0.04$ & $15.01 \pm 0.00$ & $12.76 \pm 0.05$ & 20,22 & $0.839_{-0.038}^{+0.032}$ & $0.788_{-0.032}^{+0.028}$ & $0.738_{-0.044}^{+0.039}$ & $0.756_{-0.048}^{+0.044}$ \\
\hline $0302-223$ & 1.0094 & $20.36 \pm 0.11$ & $14.67 \pm 0.04$ & $12.45 \pm 0.04$ & 18 & $0.856_{-0.044}^{+0.035}$ & $0.802_{-0.037}^{+0.032}$ & $0.758_{-0.052}^{+0.045}$ & $0.778_{-0.058}^{+0.050}$ \\
\hline $0347-383$ & 3.0250 & $20.56 \pm 0.05$ & $14.47 \pm 0.05$ & $12.23 \pm 0.12$ & $8^{g}$ & $0.841_{-0.111}^{+0.071}$ & $0.790_{-0.092}^{+0.065}$ & $0.741_{-0.126}^{+0.093}$ & $0.759_{-0.136}^{+0.102}$ \\
\hline $0405-443$ & 2.5950 & $20.90 \pm 0.10$ & $15.09 \pm 0.06$ & $12.53 \pm 0.13$ & $8^{g}$ & $0.517_{-0.205}^{+0.167}$ & $0.509_{-0.198}^{+0.150}$ & $0.377_{-0.245}^{+0.180}$ & $0.377_{-0.245}^{+0.190}$ \\
\hline $0454+039$ & 0.8597 & $20.69 \pm 0.06$ & $15.17 \pm 0.04$ & $12.33 \pm 0.09$ & 18 & $0.045_{-0.045}^{+0.192}$ & $0.045_{-0.045}^{+0.191}$ & $0.000_{-0.000}^{+0.038}$ & $0.000_{-0.000}^{+0.038}$ \\
\hline 0458-02 & 2.0400 & $21.65 \pm 0.09$ & $15.40 \pm 0.05$ & $13.13 \pm 0.02$ & 24 & $0.821_{-0.043}^{+0.037}$ & $0.773_{-0.036}^{+0.031}$ & $0.716_{-0.049}^{+0.044}$ & $0.732_{-0.054}^{+0.049}$ \\
\hline 0515-4414 & 1.1510 & $20.45 \pm 0.10$ & $14.24 \pm 0.20$ & $12.11 \pm 0.04$ & 3 & $0.905_{-0.139}^{+0.055}$ & $0.847_{-0.120}^{+0.067}$ & $0.822_{-0.168}^{+0.091}$ & $0.849_{-0.184}^{+0.091}$ \\
\hline $0528-2505$ & 2.8110 & $21.11 \pm 0.04$ & $15.47 \pm 0.02$ & $13.27 \pm 0.03$ & 1 & $0.868_{-0.023}^{+0.020}$ & $0.813_{-0.020}^{+0.018}$ & $0.774_{-0.029}^{+0.026}$ & $0.796_{-0.032}^{+0.029}$ \\
\hline $0551-3637$ & 1.9615 & $20.50 \pm 0.08$ & $15.05 \pm 0.05$ & $13.02 \pm 0.05$ & 7 & $0.939_{-0.022}^{+0.015}$ & $0.885_{-0.026}^{+0.021}$ & $0.875_{-0.036}^{+0.027}$ & $0.905_{-0.037}^{+0.026}$ \\
\hline $0812+32$ & 2.6260 & $21.35 \pm 0.10$ & $15.10 \pm 0.03$ & $13.04 \pm 0.02$ & 21 & $0.930_{-0.011}^{+0.009}$ & $0.874_{-0.013}^{+0.011}$ & $0.860_{-0.017}^{+0.016}$ & $0.889_{-0.018}^{+0.016}$ \\
\hline $0841+129$ & 2.3745 & $21.00 \pm 0.10$ & $14.87 \pm 0.04$ & $12.20 \pm 0.05$ & 1 & $0.357_{-0.105}^{+0.094}$ & $0.356_{-0.104}^{+0.091}$ & $0.187_{-0.129}^{+0.112}$ & $0.187_{-0.129}^{+0.113}$ \\
\hline $0935+417$ & 1.3726 & $20.52 \pm 0.10$ & $14.82 \pm 0.10$ & $12.25 \pm 0.01$ & 12 & $0.500_{-0.144}^{+0.124}$ & $0.493_{-0.138}^{+0.114}$ & $0.357_{-0.170}^{+0.141}$ & $0.357_{-0.171}^{+0.144}$ \\
\hline 1104-1805 & 1.6616 & $20.85 \pm 0.01$ & $14.77 \pm 0.02$ & $12.48 \pm 0.01$ & 9 & $0.804_{-0.019}^{+0.018}$ & $0.759_{-0.016}^{+0.015}$ & $0.697_{-0.021}^{+0.020}$ & $0.711_{-0.023}^{+0.022}$ \\
\hline $1117-1329$ & 3.3505 & $20.84 \pm 0.08$ & $14.82 \pm 0.05$ & $12.25 \pm 0.06$ & 14 & $0.500_{-0.110}^{+0.098}$ & $0.493_{-0.105}^{+0.090}$ & $0.357_{-0.129}^{+0.112}$ & $0.357_{-0.130}^{+0.113}$ \\
\hline $1157+0128$ & 1.9436 & $21.80 \pm 0.10$ & $15.50 \pm 0.07$ & $13.09 \pm 0.08$ & 18 & $0.690_{-0.122}^{+0.102}$ & $0.664_{-0.107}^{+0.085}$ & $0.571_{-0.136}^{+0.113}$ & $0.577_{-0.140}^{+0.120}$ \\
\hline $1209+0919$ & 2.5835 & $21.40 \pm 0.10$ & $15.22 \pm 0.04$ & $12.98 \pm 0.05$ & 21 & $0.844_{-0.054}^{+0.042}$ & $0.793_{-0.045}^{+0.037}$ & $0.744_{-0.062}^{+0.050}$ & $0.763_{-0.068}^{+0.059}$ \\
\hline $1210+1731$ & 1.8918 & $20.60 \pm 0.10$ & $14.97 \pm 0.06$ & $12.37 \pm 0.03$ & 19 & $0.458_{-0.101}^{+0.091}$ & $0.454_{-0.098}^{+0.086}$ & $0.308_{-0.121}^{+0.106}$ & $0.308_{-0.122}^{+0.107}$ \\
\hline $1215+33$ & 1.9990 & $20.95 \pm 0.07$ & $14.75 \pm 0.05$ & $12.33 \pm 0.05$ & 24 & $0.682_{-0.081}^{+0.073}$ & $0.657_{-0.071}^{+0.061}$ & $0.562_{-0.091}^{+0.080}$ & $0.567_{-0.094}^{+0.085}$ \\
\hline $1223+178$ & 2.4661 & $21.50 \pm 0.10$ & $15.16 \pm 0.02$ & $12.55 \pm 0.03$ & 19,20 & $0.450_{-0.049}^{+0.046}$ & $0.446_{-0.047}^{+0.044}$ & $0.298_{-0.058}^{+0.054}$ & $0.298_{-0.058}^{+0.055}$ \\
\hline $1253-0228$ & 2.7830 & $21.85 \pm 0.20$ & $15.36 \pm 0.04$ & $12.77 \pm 0.07$ & 21 & $0.473_{-0.121}^{+0.107}$ & $0.468_{-0.117}^{+0.099}$ & $0.326_{-0.144}^{+0.123}$ & $0.326_{-0.145}^{+0.124}$ \\
\hline $1328+307$ & 0.6922 & $21.25 \pm 0.02$ & $14.95 \pm 0.15$ & $12.72 \pm 0.12$ & 11 & $0.852_{-0.172}^{+0.087}$ & $0.799_{-0.144}^{+0.086}$ & $0.753_{-0.194}^{+0.121}$ & $0.773_{-0.208}^{+0.131}$ \\
\hline $1331+170$ & 1.7764 & $21.18 \pm 0.04$ & $14.62 \pm 0.00$ & $12.54 \pm 0.03$ & 24 & $0.925_{-0.010}^{+0.009}$ & $0.869_{-0.011}^{+0.010}$ & $0.853_{-0.016}^{+0.014}$ & $0.882_{-0.017}^{+0.015}$ \\
\hline $1351+318$ & 1.1491 & $20.23 \pm 0.10$ & $14.74 \pm 0.09$ & $12.52 \pm 0.13$ & 24 & $0.856_{-0.132}^{+0.074}$ & $0.802_{-0.110}^{+0.072}$ & $0.758_{-0.151}^{+0.102}$ & $0.778_{-0.164}^{+0.111}$ \\
\hline $1354+258$ & 1.4200 & $21.54 \pm 0.06$ & $15.03 \pm 0.09$ & $12.59 \pm 0.13$ & 24 & $0.658_{-0.196}^{+0.152}$ & $0.636_{-0.179}^{+0.128}$ & $0.535_{-0.222}^{+0.170}$ & $0.539_{-0.226}^{+0.180}$ \\
\hline $1451+1223$ & 2.2550 & $20.30 \pm 0.15$ & $14.33 \pm 0.07$ & $11.85 \pm 0.11$ & 4 & $0.612_{-0.167}^{+0.139}$ & $0.596_{-0.155}^{+0.119}$ & $0.484_{-0.192}^{-0.222}$ & $0.487_{-0.194}^{+0.220}$ \\
\hline 2206-199A & 1.9200 & $20.65 \pm 0.10$ & $15.32 \pm 0.02$ & $12.91 \pm 0.01$ & 23 & $0.699_{-0.021}^{+0.021}$ & $0.671_{-0.018}^{+0.017}$ & $0.580_{-0.024}^{+0.023}$ & $0.586_{-0.025}^{+0.024}$ \\
\hline $2230+025$ & 1.8642 & $20.85 \pm 0.08$ & $15.18 \pm 0.02$ & $12.80 \pm 0.03$ & 24 & $0.717_{-0.034}^{+0.032}$ & $0.687_{-0.028}^{+0.026}$ & $0.601_{-0.037}^{-0.024}$ & $0.608_{-0.039}^{+0.023}$ \\
\hline $2231-0015$ & 2.0662 & $20.56 \pm 0.10$ & $14.66 \pm 0.07$ & $12.35 \pm 0.03$ & 24 & $0.787_{-0.065}^{+0.055}$ & $0.745_{-0.054}^{+0.046}$ & $0.678_{-0.073}^{+0.063}$ & $0.690_{-0.078}^{+0.069}$ \\
\hline $2243-6031$ & 2.3300 & $20.67 \pm 0.02$ & $14.92 \pm 0.03$ & $12.22 \pm 0.03$ & 10 & $0.310_{-0.072}^{+0.067}$ & $0.309_{-0.072}^{+0.066}$ & $0.129_{-0.089}^{+0.081}$ & $0.129_{-0.089}^{+0.081}$ \\
\hline 2314-409 & 1.8573 & $20.90 \pm 0.10$ & $15.08 \pm 0.10$ & $12.52 \pm 0.10$ & 5 & $0.513_{-0.206}^{+0.168}$ & $0.506_{-0.199}^{+0.151}$ & $0.372_{-0.246}^{+0.189}$ & $0.373_{-0.247}^{+0.193}$ \\
\hline $2342+3417$ & 2.9084 & $21.10 \pm 0.10$ & $14.98 \pm 0.06$ & $12.50 \pm 0.11$ & 21 & $0.611_{-0.161}^{+0.134}$ & $0.595_{-0.149}^{+0.1159}$ & $\begin{array}{r}-0.483_{-0.184}^{+0.246} \\
\end{array}$ & $0.486_{-0.185}^{+0.247}$ \\
\hline 2359-0216 & 2.0950 & $20.70 \pm 0.10$ & $14.54 \pm 0.03$ & $12.60 \pm 0.03$ & 24 & $0.956_{-0.007}^{+0.006}$ & $0.908_{-0.011}^{+0.009}$ & $0.905_{-0.014}^{+0.012}$ & $0.933_{-0.013}^{+0.010}$ \\
\hline
\end{tabular}

${ }^{a}$ The fraction in dust of iron is derived from the expression (2) and represents a dust-to-metal ratio by number. In the $z=0.8597$ system towards QSO $0454+039$ the measured $[\mathrm{Zn} / \mathrm{Fe}]$ ratio is lower than the adopted $[\mathrm{Zn} / \mathrm{Fe}]_{\mathrm{g}}$ for the parameter sets E0 and E1; in these cases we set $f_{\mathrm{Fe}}=0$ to avoid a negative, unphysical result. For the same reason, the error bars of $f_{\mathrm{Fe}}$ have been truncated in a few cases.

${ }^{b}$ Logarithm of the observed column densities expressed in atoms $\mathrm{cm}^{-2}$.

${ }^{c}$ Parameter set $\mathrm{Sa}:[\mathrm{Zn} / \mathrm{Fe}]_{\mathrm{g}}=0.0$ dex, $f_{\mathrm{Zn}, w d}=0.59$; results independent of the adopted value of $\varepsilon_{\mathrm{Zn}}$.

${ }^{d}$ Parameter set $\mathrm{Sb}:[\mathrm{Zn} / \mathrm{Fe}]_{\mathrm{g}}=0.0 \mathrm{dex}, f_{\mathrm{Zn}, w d}=0.30$; results independent of the adopted value of $\varepsilon_{\mathrm{Zn}}$.

${ }^{e}$ Parameter set E0: $[\mathrm{Zn} / \mathrm{Fe}]_{\mathrm{g}}=+0.1 \mathrm{dex}, f_{\mathrm{Zn}, w d}=0.59 ; \varepsilon_{\mathrm{Zn}}=0$.

${ }^{f}$ Parameter set E1: $[\mathrm{Zn} / \mathrm{Fe}]_{\mathrm{g}}=+0.1 \mathrm{dex}, f_{\mathrm{Zn}, w d}=0.59 ; \varepsilon_{\mathrm{Zn}}=1$.

${ }^{g}$ Sum of all components of the Fe II and Zn II column densities.

References - (1) Centurión et al. (2003; (2) Centurión et al. (2004) (work in preparation); (3) de la Varga et al. (2000); (4) Dessauges-Zavadsky et al. (2003); (5) Ellison \& Lopez (2001); (6) Ge \& Bechtold (1997); (7) Ledoux et al. (2002); (8) Ledoux et al. (2003); (9) Lopez et al. (1999); (10) Lopez et al. (2002); (11) Meyer \& York (1992); (12) Meyer et al. (1995); (13) Molaro et al. (2000); (14) Péroux et al. (2002); (15) Petitjean et al. (2000); (16) Pettini et al. (1994); (17) Pettini et al. (1999); (18) Pettini et al. (2000); (19) Prochaska et al. (2001) (I database); (20) Prochaska et al. (2002) (IV database); (21) Prochaska et al. (2003) (ESI database); (22) Prochaska \& Wolfe (1996); (23) Prochaska \& Wolfe (1997); (24) Prochaska \& Wolfe (1999). 

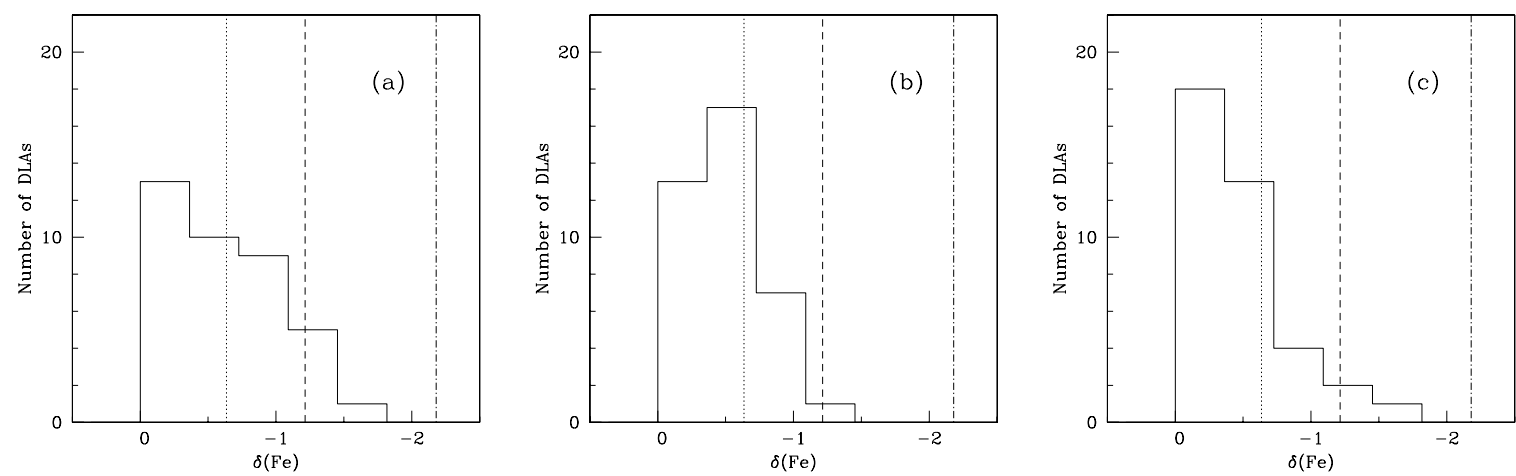

Fig. 1. Distribution of iron depletions for the DLA systems in Table 1. Panels a), b) and c) correspond to cases Sa, Sb and E1, respectively. Vertical lines: typical values of interstellar iron depletions in the Milky Way (dotted line: warm halo gas; dashed line: warm disk gas; dotteddashed line: cold disk gas).
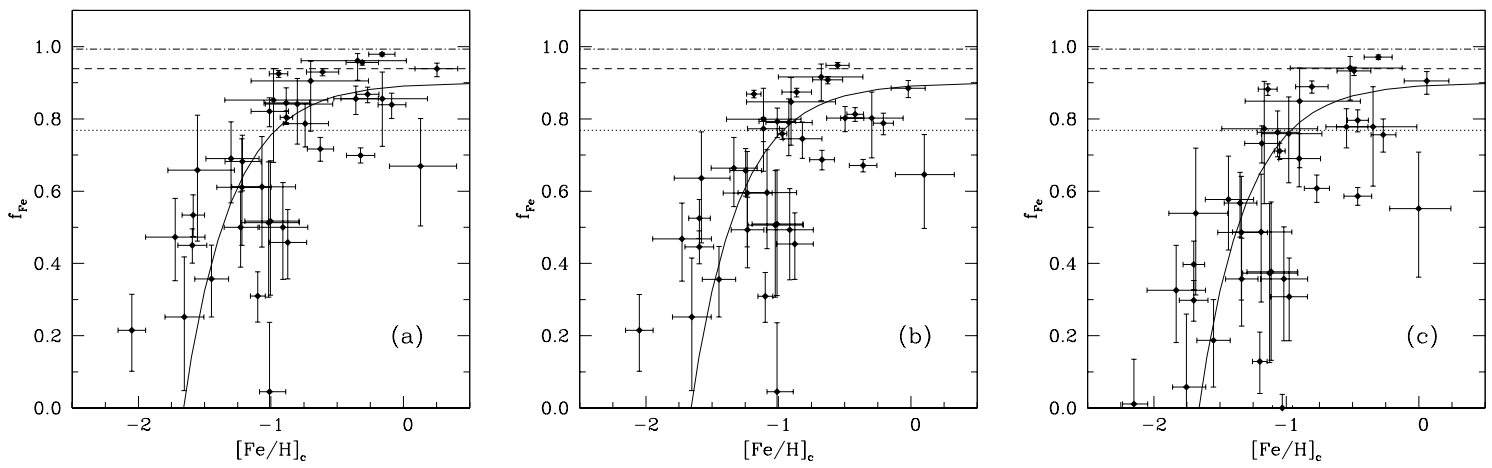

Fig. 2. Iron dust fraction versus metallicity for the sample of DLA systems listed in Table 1. Panels a), b) and c) correspond to cases Sa, $\mathrm{Sb}$ and E1, respectively. Horizontal lines: representative values of iron dust fractions in the Galactic ISM (legenda as in Fig. 1). Solid curve: empirical law (4) describing the evolution of the dust fraction with metallicity.

One can see that, as a consequence, the rise of $f_{\mathrm{Fe}}$ with metallicity would be even steeper. We now discuss the stability of the result in the light of possible observational bias.

\subsection{Selection bias}

Several types of observational bias are known to affect the measurements of the $N(\mathrm{HI}), N(\mathrm{Fe} I \mathrm{I})$, and $N(\mathrm{Zn} \mathrm{II})$ column densities. For instance, the detection limit ${ }^{3}$ of the strongest line of the $\mathrm{Zn}$ II resonance doublet prevents the measurement of column densities $N(\mathrm{Zn}$ II $) \lesssim 10^{11.75}$ atoms $\mathrm{cm}^{-2}$. The detection limit of Fe II depends on the wavelength coverage of the spectra, since a variety of strong Fe II transitions are present at different wavelengths. Assuming that we do not cover the stronger transitions at longer rest wavelengths, we obtain a conservative limit from the detection limit of the $160.8 \mathrm{~nm}$ line, $N(\mathrm{Fe}$ II $) \lessgtr 10^{12.88}$ atoms $\mathrm{cm}^{-2}$. Also H I measurements are affected by a selection bias. The range of $N(\mathrm{H} \mathrm{I})$ is limited on the one side by the definition threshold in DLA systems $N(\mathrm{HI}) \geq$ $10^{20.3}$ atoms cm $\mathrm{cm}^{-2}$ and, on the other side, by the fact that values above $N(\mathrm{H} \mathrm{I})>10^{20.85}$ atoms $\mathrm{cm}^{-2}$ have never been observed,

\footnotetext{
3 The detection limits are estimated at a $3 \sigma$ level for a signal-tonoise ratio $S / N \simeq 30$ and resolving power $R=\lambda /(\Delta \lambda) \simeq 5 \times 10^{4}$ at $\lambda \simeq 500 \mathrm{~nm}$. Oscillator strengths for the $\mathrm{Zn}$ II $202.6136 \mathrm{~nm}$ and the Fe II $160.8451 \mathrm{~nm}$ lines are taken from Bergeson \& Lawler (1993) and from Welty et al. (1999), respectively.
}

even if they are detectable. Finally, the obscuration of the background QSO by dust extinction in the DLA system could be responsible for the lack of systems with high metal column densities, namely those with $N(\mathrm{Zn} \mathrm{II}) \gtrsim 10^{13.1}$ atoms $\mathrm{cm}^{-2}$ (Boissé et al. 1998), which are also detectable.

To assess the effect of these observational biases on the present study we built a mock sample of $n$ DLA systems with a flat distribution of metallicities and depletions (or dust fractions). We then assigned to each system a random-generated HI column density in the allowed range $20.3 \leq \log N(\mathrm{HI}) \leq$ 20.85 and computed $N(\mathrm{Fe} I \mathrm{II})$ and $N(\mathrm{Zn}$ II) for each pair of $\left([\mathrm{Fe} / \mathrm{H}], f_{\mathrm{Fe}}\right)$ values. Finally, we rejected cases with $N(\mathrm{Zn} \mathrm{II})$ and $N(\mathrm{Fe}$ II) outside the limits discussed above. At this point, we applied to the biased sample the same method for the derivation of dust fractions and metallicities described in Sect. 2.1 and the same analysis presented in Sects. 3.1 and 3.2. In this way, we tested the potential effects of the column-density bias and, at the same time, of the application of the method itself. The results are shown in Fig. 3 for $n=1000$ systems (before rejection).

The frequency distribution of depletions of the biased mock sample is shown in panel (a) of the figure. In spite of the large fraction of data points rejected (65\%), the distribution is still approximately flat in the range $-2.2 \leq \delta(\mathrm{Fe}) \leq 0$, as defined by construction. This indicates that the overall distribution of depletions is not significantly affected by bias and, therefore, the 

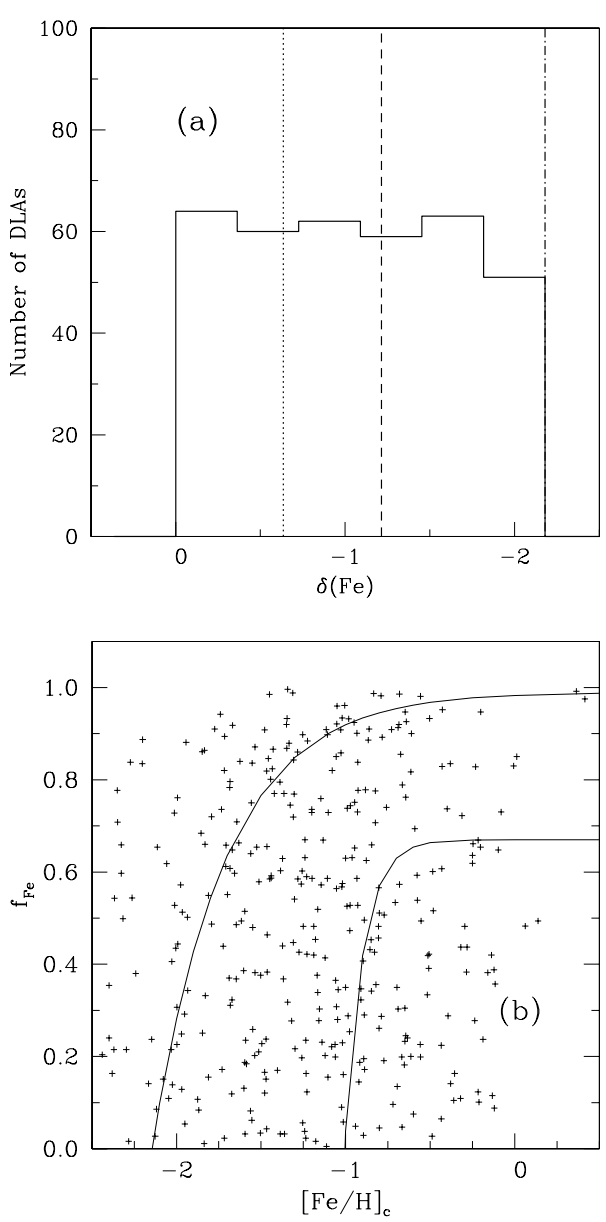

Fig. 3. Test of selection effects performed on a mock sample of $n=$ 1000 DLA systems with H I, Fe II and Zn II column densities rejected as explained in Sect. 3.3. a) Frequency distribution of iron depletion (after rejection) for an adopted flat distribution of $\delta(\mathrm{Fe})$. b) Dust fraction versus metallicity for an adopted flat distribution of $f_{\mathrm{Fe}}$; the solid curves represent the envelopes of the real data in Fig. 2. The depletions of the mock sample have been modeled with the set of parameters Sa (Table 1); similar results are obtained for the other cases.

steepness of the real distribution in Fig. 1 is genuine. We conclude that the deficiency of systems with high levels of dust depletion seems to be an intrinsic property of DLA systems rather than a consequence of selection effects.

The plot of dust fraction versus metallicity, for the mock sample built to uniformly cover the range $0 \leq f_{\mathrm{Fe}} \leq 1$, is shown in Fig. 3b. The non-rejected data of the mock sample (crosses in the figure) are able to populate regions outside the boundaries in which the real data are confined (solid curves). This indicates that the selection bias is not responsible for the lack of real data outside these boundaries. In addition, the rather homogeneous distribution of the data in Fig. $3 b$, derived from the application of our method to the mock column densities, indicates that the method itself does not induce artificial trends between $f_{\mathrm{Fe}}$ and $[\mathrm{Fe} / \mathrm{H}]$. We conclude that the rise of dust fraction with increasing metallicity is a genuine property of DLA systems.

Clearly, a better understanding of the dust obscuration effect is required to take into account the role of selection bias in a more realistic way. In any case, we expect the extinction of the QSO to be more effective with increasing metallicity of the intervening absorbers. Therefore, the fact that DLA systems with high dust content $\left(f_{\mathrm{Fe}} \gtrsim 0.8\right)$ are detected at high metallicity demonstrates that the obscuration bias cannot be responsible for the lack of such systems at low metallicity (compare the data points with $[\mathrm{Fe} / \mathrm{H}] \lesssim-1.5 \mathrm{dex}$ and $\gtrsim-1.5 \mathrm{dex}$ in Fig. 2). On the other hand, the number of absorbers with depletions typical of cold interstellar gas and high metallicity can be affected by dust obscuration. Therefore the deficit of cold gas depletions could be less severe than shown in Fig. 1, but only for systems of high metallicity.

\subsection{Time evolution}

Finally we study the time evolution of the dust fraction after converting the redshift of each absorber to the look-back time or age of the universe ${ }^{4}$. The advantage of this approach is that we compare $f_{\mathrm{Fe}}$ with a completely independent observable, while in the study versus metallicity we compare quantities derived from the measurements of the same column densities. However, the interpretation of the time evolution is complicated by the fact that DLA galaxies may start their chemical evolution at different cosmic times. Inspection of the plot of $f_{\mathrm{Fe}}$ versus age of the Universe, shown in Fig. 4a, does not reveal a clear trend, even though the data are consistent with a sudden rise of the dust-to-metal ratio between $\approx 2$ and $4 \mathrm{Gyr}$ after the Big Bang. Some evidence of evolution is found by binning all the systems seen before and after the median value of look-back time of the present sample, $t_{1} \simeq 10.5 \mathrm{Gyr}$, and comparing the frequency distribution of their depletions (Fig. 4b). One can see that the two distributions are slightly shifted, the DLAs seen at the earliest age of the universe (shaded histogram) having lower depletions $|\delta(\mathrm{Fe})|$ than those observed in more recent times (empty hystogram). Again, these results are valid for all the possible choices of input parameters (case $\mathrm{Sa}$, shown in the figure, and cases Sb, E0 and E, not shown).

\section{Discussion}

We now discuss the empirical results presented in the previous section assuming that they are substantially unaffected by selection bias. We first try to understand whether the observed trends are mainly governed by variations of the physical properties or by chemical evolution. An example of the difficulty in disentangling these two effects is given in Fig. 5a, where we plot the iron depletions versus $l_{\mathrm{c}} \propto N\left(\mathrm{C} \mathrm{II}^{*}\right) / N(\mathrm{HI})$, an indicator of the cooling rate of the gas (Wolfe et al. 2003a). Apparently, $|\delta(\mathrm{Fe})|$ seems to increase with $\log l_{\mathrm{c}}$ and this trend could be taken as evidence for a correlation of the depletions with some physical property of the gas. However, $l_{\mathrm{c}}$ is by definition proportional to the metallicity and the trend in Fig. 5a may simply mirror the rise of $f_{\mathrm{Fe}}$ with metallicity shown in Fig. 2. We now consider other observational evidence that might help in disentangling the role of physical processes.

4 We calculated these conversions for a flat universe with $H_{0}=$ $71 \mathrm{~km} \mathrm{~s}^{-1} \mathrm{Mpc}^{-1}$ and $\Omega_{m}=0.27$ (Spergel et al. 2003). 

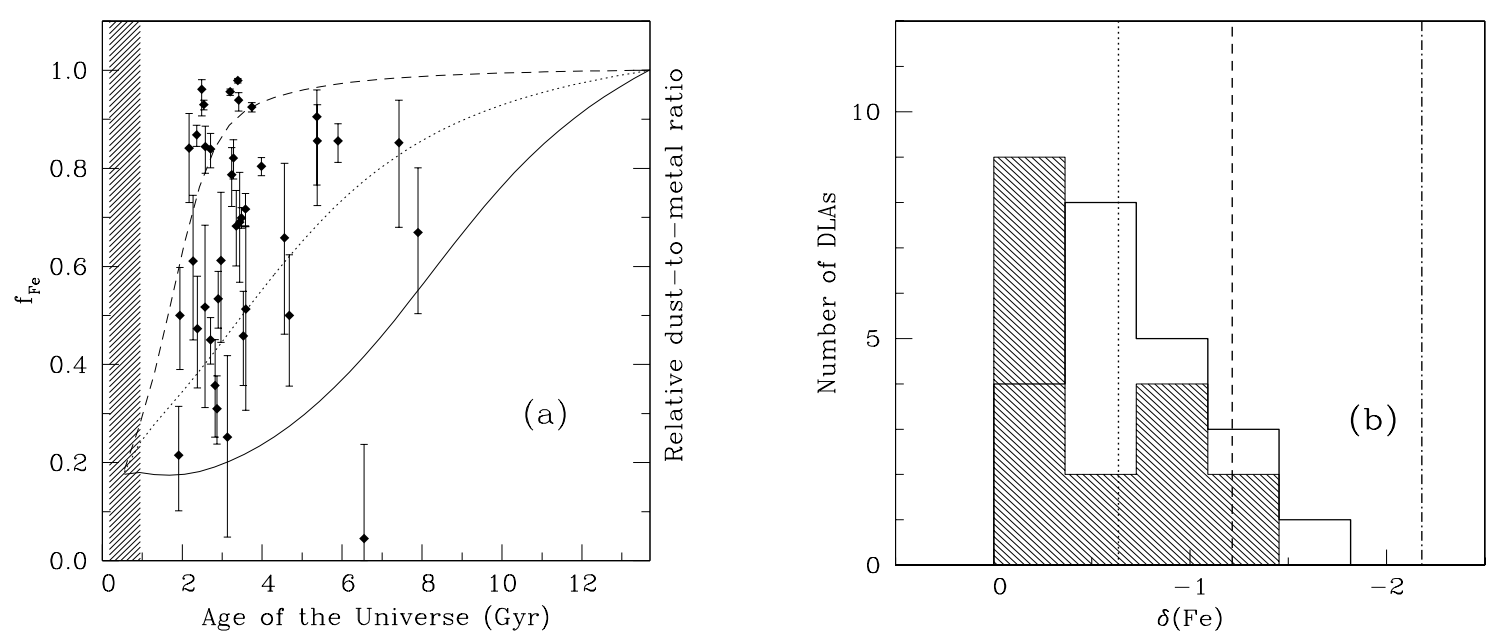

Fig. 4. Time evolution of the dust content in DLA systems. a) Diamonds: $f_{\mathrm{Fe}}$ versus age of the universe; dashed area: reionization epoch $6 \leq z \leq 20$ (Fan et al. 2003; Spergel et al. 2003); solid, dotted and dashed curves: Inoue's (2003) model predictions of the evolution of the relative dust-to-metal ratio for different sets of input parameters (see text for explanations); the curves have been normalized to the present-day value and scaled assuming a formation redshift $z_{f}=20$. b) Frequency distribution of iron depletions in two bins of look-back time (shaded area: $t_{1} \geq 10.5 \mathrm{Gyr}$; empty histogram: $t_{1}<10.5 \mathrm{Gyr}$ ). The fraction in dust are computed for the set of parameters Sa.

\subsection{The dependence on physical parameters}

In the Galactic ISM, each interstellar phase is characterized by a particular level of depletion. Cold, high-density gas, where the dust has higher accretion probability and is more shielded from destruction processes, is characterized by high depletions; warm, low-density gas is instead known to have low depletions (e.g., Savage \& Sembach 1996). This link between the physical state and level of depletion might be at work also in the ISM of other galaxies, including DLA systems. If so, low values of depletions would map regions of warm gas, while higher depletions colder regions. According to this interpretation, the frequency distribution in Fig. 1 would indicate that in DLA systems warm regions are more frequent (have a higher cross-section) than cold regions. The evolutionary trends with metallicity (Fig. 2) and cosmic time (Fig. 4) would indicate that cold regions are extremely rare in the early stages of evolution, but eventually they become more frequent.

Some evidence in favour of this interpretation comes from measurements of the spin temperature, $T_{\mathrm{s}}(\mathrm{K})$, derived $21-\mathrm{cm}$ absorption observations (Carilli et al. 1996; Chengalur \& Kanekar 2000; Kanekar \& Chengalur 2003) and from studies of the $N(\mathrm{C}$ II $) / N(\mathrm{C}$ I) ratio (Liszt 2002), which suggest that the warm phase is generally dominant in DLA systems, especially at high redshift (see however Wolfe et al. 2003b). Nevertheless, a detailed study of the results on a system-by-system basis does not support a direct connection between depletions and physical parameters in DLAs. In fact, if the observed trends of depletions were governed by variations of the physical properties, we would expect to find a decrease of $|\delta(\mathrm{Fe})|$ with increasing $T_{\mathrm{s}}$ or $N(\mathrm{C} \mathrm{II}) / N(\mathrm{C} \mathrm{I})$, i.e. with increasing importance of the warm component. The comparison of depletions versus $T_{\mathrm{s}}$ for the systems in common with the compilation by Kanekar \& Chengalur (2003) does not support this expectation (Fig. 5b). Also the comparison with the $N(\mathrm{C}$ II $) / N(\mathrm{C}$ I $)$ determinations by Liszt (2002) does not reveal the expected trend (Fig. 5c).
We also compared the depletions with the molecular fraction $f\left(\mathrm{H}_{2}\right)=2 N\left(\mathrm{H}_{2}\right) /\left[N(\mathrm{HI})+2 N\left(\mathrm{H}_{2}\right)\right]$, which is sensitive to physical conditions. Some evidence for a trend between differential depletions and $f\left(\mathrm{H}_{2}\right)$ has been reported in previous work (Levshakov et al. 2000; Ledoux et al. 2003). The limited number of measurements in common with the present sample are marginally consistent with the existence of such a trend (Fig. 5d). This trend would only confirm the key role of dust in the process of $\mathrm{H}_{2}$ formation, but would not provide evidence for a direct link between the physical properties and the abundance of dust. In fact, the correlation is expected because dust acts as a catalyst of $\mathrm{H}_{2}$ formation and provides a shield against photo-dissociation of molecules by ultraviolet radiation.

In summary, there is no evidence that the evolution of the dust fractions/depletions is primarily governed by variations of the physical state of the gas. In the following we consider the chemical evolution as the main driver of the evolution of the dust abundance in DLA systems. However, we expect that variations of the physical state of the gas will contribute to the scatter of dust abundance observed at a given metallicity.

\subsection{The dependence on chemical evolution}

The regular trend shown by the data in Fig. 2 is an argument in favour of a direct dependence of depletion on the metallicity, i.e. on the chemical evolution.

The rise of the dust-to-metal ratio $f_{\mathrm{Fe}}$ in the course of chemical evolution is unexpected, given the approximately constant dust-to-metal ratio $\mathcal{D} / \mathcal{Z}$ measured in local galaxies (Issa et al. 1990; Schmidt \& Boller 1993). However, the two results are not in contradiction since the data in Fig. 2 suggest the existence of a flattening of $f_{\mathrm{Fe}} \approx \mathcal{D} / \mathcal{Z}$ at $[\mathrm{Fe} / \mathrm{H}] \gtrsim-1$ dex, i.e. when the metallicity approaches values typical of the local sample of $\mathcal{D} / \mathcal{Z}$ measurements. 

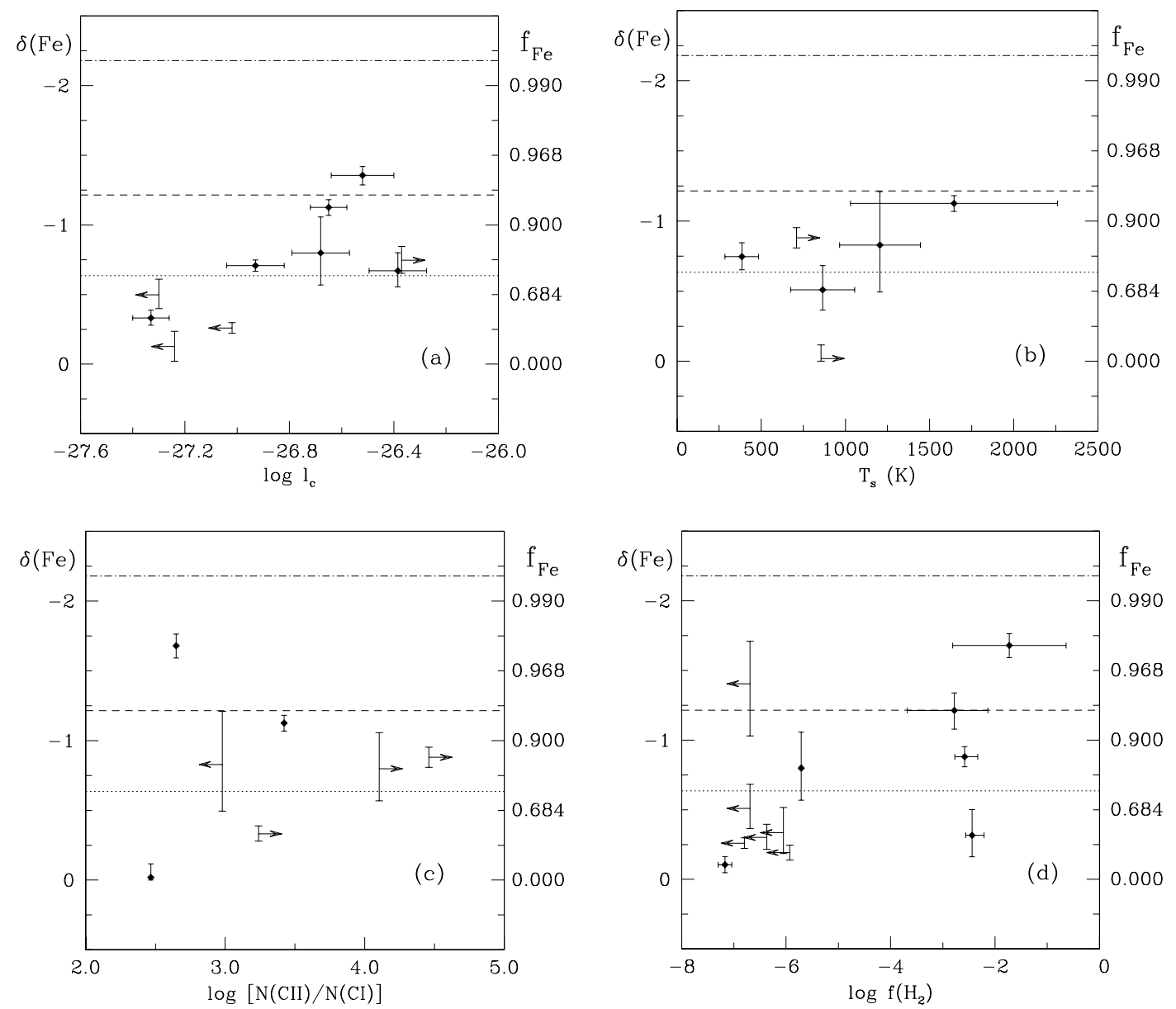

Fig. 5. Iron depletions/dust fractions versus some physical parameters measured in DLA systems. a) Cooling rate per $\mathrm{H}$ atom, $l_{\mathrm{c}}$ (Wolfe et al. 2003a). b) Spin temperature, $T_{\mathrm{s}}$ (Kanekar \& Chengalur 2003). c) $N(\mathrm{C}$ II $) / N(\mathrm{C} \mathrm{I})$ ionization ratio (Liszt 2002). d) Molecular fraction $f\left(\mathrm{H}_{2}\right)$ (Ledoux et al. 2003). Depletions are computed in case Sa. Horizontal lines as in Fig. 1.

Also the scatter of $f_{\mathrm{Fe}}$ in DLA systems is consistent with the observed properties of the $\mathcal{D} / \mathcal{Z}$ ratio in present-day galaxies. In fact, a spread of the $\mathcal{D} / \mathcal{Z}$ ratio is found when different types of galaxies are compared, such as the blue compact dwarfs and dwarf irregulars investigated by Lisenfeld \& Ferrara (1998). Therefore, the scatter of $f_{\mathrm{Fe}}$ may simply reflect the inhomogeneity of the population of DLA systems, which in fact is believed to include galaxies of different types. The $\mathcal{D} / \mathcal{Z}$ ratio about $1 / 4$ to $1 / 7$ of the Galactic one recently found in a dwarf galaxy with an average metallicity $\simeq 1 / 4$ solar (Galliano et al. 2003) is a further example indicating that the properties of the dust fraction $f_{\mathrm{Fe}}$ in DLA systems are consistent with the properties of the dust-to-metal ratios $\mathcal{D} / \mathcal{Z}$ in the local universe. Thefore, the trend seen in Fig. 2 may represent a general characteristic of galactic evolution, rather than a specific property of DLA galaxies.

The rise of $\mathcal{D} / \mathcal{Z}$ with metallicity suggests that the efficiency of dust formation is very low at the earliest stages of chemical evolution but increases regularly as metals become more abundant. As a first step in interpreting this effect, we consider the possibility that the most common processes of dust formation may show a dependence on the metallicity. Dust can be formed in the ejecta of type II SNe (Moseley et al. 1989; Elmhamdi et al. 2003; Dunne et al. 2003) and in the cool winds of late-type giant stars (see Sedlmayr 1989, and Refs. therein). It can probably also be formed in the ejecta of type Ia SNe, even though in this case the evidence is not direct (Clayton et al. 1997). Other processes of dust formation are believed to give a minor contribution on a galactic scale (see e.g., Dwek 1998). The composition of the dust is poorly known on observational grounds and often inferred from chemical equilibrium condensation calculations. The relative abundance of iron in dust grains is not known, even though iron could be present both in carbon-rich dust (e.g., $\mathrm{Fe}_{3} \mathrm{C}$; Lattimer et al. 1978), and in silicate grains (e.g., $\mathrm{FeSiO}_{3}, \mathrm{Fe}_{2} \mathrm{SiO}_{4}$; Ossenkopf et al. 1992); the dust formed by SNe Ia, if any, is expected to be iron-rich. In the following we consider the possible dependence on the metallicity of the main mechanisms of dust formation, assuming that a significant fraction of iron can be incorporated in dust form in such processes.

Given the short life-times of their massive progenitors, SNe II probably represent the only source of dust at very high redshift. The time-scale for dust production by the winds of red giants or by SNe Ia is of the order of $1 \mathrm{Gyr}$, i.e. larger than the age of the universe at $z \gtrsim 4$. The existence of dust at $z>4$ (e.g., Carilli et al. 2000) is a stringent argument in favour of the production of dust by SNe II in the early universe. The systems with highest redshift in our sample are seen at about $\gtrsim 1.8 \mathrm{Gyr}$ 
after the start of the reionization epoch (Fig. 4a) and a significant part of their dust must have been produced by SNe II. A dust formation mechanism for SNe II dependent on metallicity has been found by Todini \& Ferrara (2001). In this process the density of heavy elements in the ejecta becomes large enough at higher metallicities to allow the state of supersaturation to be reached more easily, favouring the production of dust. The metallicity-dependent dust formation in SNe II found by Todini \& Ferrara, together with the lack of other sources of dust, may explain the very low dust fraction typical of most of the highestredshift systems in our sample (shaded histogram in Fig. 4b), which have the lowest metallicities.

After $\approx 1$ Gyr from the start of the chemical evolution the dust starts to be injected also by the winds of red giants and possibly by SNe Ia. We consider three possibilities: (1) these additional contributions are negligible compared to the dust production of SNe II; (2) the production of dust in the wind of late-type giants is dominant; and (3) the production by SN Ia is dominant.

In the first case the metallicity-dependent dust production of SNe II may explain the rise of the dust fraction with metallicity not only at the highest redshifts, but also at later stages of evolution.

In the second case another mechanism may provide a metallicity-dependent dust formation, namely the dependence on the metallicity of the wind efficiency. In fact, recent work on dust production in giant stars indicates that the efficiency of the stellar winds is lower at lower metallicity (Ferrarotti \& Gail 2003). Therefore, the gradual rise of the efficiency of their winds with increasing metallicity may yield a gradual rise of the dust fraction. However, this mechanism can explain the rise of $f_{\mathrm{Fe}}$ only if iron is already present in the atmosphere of the late-type giants, since iron is not synthesized by these stars. This implies that this mechanism could only work at later stages of evolution, $\approx 1$ Gyr after iron is already present in the gas out of which the future late-giants are born.

In the third case, the (hypothetical) iron-rich dust from SN Ia could provide an additional interpretation: the time delay between the early production of dust by SNe II and the subsequent production of iron-rich dust by SNe Ia would work as a mechanism for increasing the iron dust fraction with time. A potential problem with this explanation is the possibility that SNe Ia can only explode when $[\mathrm{Fe} / \mathrm{H}] \gtrsim-1$ dex (Kobayashi et al. 1998), i.e. after the rapid increase of $f_{\mathrm{Fe}}$ seen in Fig. 2.

In summary, all the important mechanisms of dust formation might be able to provide a rise of the dust fraction in the course of chemical evolution. The most convincing mechanism, however, is the metallicity-dependent production by SNe II proposed by Todini \& Ferrara (2001). Clearly, the evolution of the dust content will be determined not only by the dust formation rate, but also by the destruction and accretion rates, not discussed here in detail. By assuming that the ratio of the destruction and accretion time-scales is proportional to the time-scale of star formation, Inoue (2003) predicts that the dust-to-metal ratio should increase in the course of chemical evolution. The rise of the dust-to-metal ratio predicted by the "standard Galactic model" of Inoue (solid curve in Fig. 4a) is less steep than the rise observed in DLA systems. The better agreement with the case of "no SNe destruction" (dotted line) may simply reflect the fact that iron is one of the most refractory elements, not easily destroyed by the SNe shocks. The range of dust-to-metal ratios predicted by the three models shown in the Fig. 6 of Inoue (2003) approximately bracket the observed data. The highest values of $f_{\mathrm{Fe}}$ observed at $\approx 2 \mathrm{Gyr}$ after the Big Bang suggest a very early epoch of galaxy formation for these particular DLA systems, since even the model with the highest accretion rate (dashed line) has some difficulty in reproducing these high values of $f_{\mathrm{Fe}}$, even assuming a formation redshift $z_{f}=20$.

The above conclusions must be considered tentative given the simplified nature of the analytical models and because the conversion between $f_{\mathrm{Fe}}$ and the dust-to-metal ratio by mass is model-dependent (e.g. the conversion requires some assumption on the dust composition). Nevertheless, this exercise is an example that shows how the present data can be used to constrain galactic evolution models incorporating the cycle of dust formation and destruction. The implementation of the dust component in realistic models calibrated on galaxies of the local universe can in turn be used to provide fresh clues on the redshift of formation and the nature of DLA galaxies (e.g., Calura et al. 2003).

\section{Conclusions}

We have investigated the evolutionary properties of the dustto-metal ratio in DLA systems with the aim of casting light on the early build-up of dust in galaxies. The results that we obtain are representative of high-redshift, metal-poor galaxies selected on the basis of their $\mathrm{H}$ I absorption column density, i.e. $N(\mathrm{H} \mathrm{I}) \geq 2 \times 10^{20}$ atoms cm $\mathrm{cm}^{-2}$.

As an estimator of the dust-to-metal ratio in DLA systems we have used the fraction of iron atoms in dust form, $f_{\mathrm{Fe}}$. One advantage of this approach is that iron is a refractory element which traces the presence of dust even in harsh interstellar environments. The dust fraction $f_{\mathrm{Fe}}$ has been derived by comparing the iron abundance with the abundance of the volatile element $\mathrm{Zn}$. As in Paper I, we take into account the fact that also $\mathrm{Zn}$ can be incorporated in dust form. However, the methodology adopted here features important improvements over the one followed in Paper I: (1) the intrinsic $\mathrm{Zn} / \mathrm{Fe}$ ratio in DLA systems is now a free input parameter; (2) the dust chemical composition is allowed to vary in different galactic environments (see Paper II); (3) we prefer the dust-to-metal ratio, rather than the less reliable dust-to-gas ratio, to study the evolution of the dust content. The main results of the present study can be summarized as follows.

The frequency distribution of iron depletions, $\delta(\mathrm{Fe})=$ $\log \left(1-f_{\mathrm{Fe}}\right)$, peaks at $\delta(\mathrm{Fe}) \sim 0$, and decreases regularly with increasing $|\delta(\mathrm{Fe})|$. A significant fraction of DLA systems has depletions lower than the lowest values measured in the Galactic ISM, i.e. those of the Galactic halo gas. The frequently quoted claim that depletions of DLA systems "are typical of Galactic warm gas" is correct only for the remaining systems. High values of depletion, typical of Galactic cold disk gas, are not found. 
The dust fraction $f_{\mathrm{Fe}}$ increases with metallicity, albeit with a significant scatter and a flattening of the trend when $[\mathrm{Fe} / \mathrm{H}] \gtrsim$ -1 dex. An empirical relation between dust fraction and metallicity of the type $f_{\mathrm{Fe}} \approx f_{\mathrm{Fe}, \circ}-10^{\alpha\left([\mathrm{Fe} / \mathrm{H}]-[\mathrm{Fe} / \mathrm{H}]_{\circ}\right)}$, with $f_{\mathrm{Fe}, \circ} \simeq 0.9$, $\alpha \simeq-1.2$, and $[\mathrm{Fe} / \mathrm{H}]_{\circ} \simeq-1.7$, provides a good fit to the data.

The comparison between the dust fraction $f_{\mathrm{Fe}}$ and the lookback time, $t_{1}$ (derived from the absorption redshift), reveals weak evidence for a rise of $f_{\mathrm{Fe}}$ with cosmic time. In spite of a large scatter, the data are consistent with a fast rise of $f_{\mathrm{Fe}}$ starting at about 2 Gyr after the Big Bang and lasting a few billion years. Indeed, the comparison of the frequency distributions of depletions for the two sub-samples with $t_{1} \geq 10.5 \mathrm{Gyr}$ and $t_{1}<10.5$ Gyr suggests that the dust fraction does increase with cosmic time.

The above conclusions are rather stable for different choices of the input parameters required for the determination of $f_{\mathrm{Fe}}$. In particular, they are not affected by changes of the adopted guess of the intrinsic $\mathrm{Zn} / \mathrm{Fe}$ ratio in DLA systems, $[\mathrm{Zn} / \mathrm{Fe}]_{\mathrm{g}}$, in line with the measurements of the same ratio in metal-poor stars. The large number of measurements recently reported by three independent groups consistently indicate that the $\mathrm{Zn} / \mathrm{Fe}$ ratio in metal-poor stars is very close to solar and probably constant in the metallicity interval typical of DLA systems. Assuming that the intrinsic $\mathrm{Zn} / \mathrm{Fe}$ ratio decreases with chemical evolution, as reported by some authors, the existence of the trends with metallicity and cosmic time would even be reinforced.

We have tested the robustness of the results in light of the selection biases that are known to affect the measurements of the H I, Fe II, and Zn II column densities. From the analysis of a mock sample of DLA systems with assigned values of dust fraction and metallicity we conclude that the bias effects are small and therefore that the trend between dust fraction and metallicity represents a genuine property of DLA systems. This is also true for the distribution of depletions, which peaks at low values and shows a deficiency of systems with depletions typical of Galactic cold disk gas. The dust obscuration bias may in part be responsible for such a deficiency, but probably only for systems of high metallicity.

The presence of different interstellar phases with a range of physical parameters in DLA systems may contribute to the scatter of the dust fractions measured at each metallicity. However, the depletions do not show correlations with physical parameters measured in DLA systems, such as the spin temperature or the $\mathrm{CII} / \mathrm{CI}$ ionization ratio. We propose that the evolutionary trends of the dust fraction in DLA systems are mostly governed by the chemical evolution of the galaxies where the absorptions originate, rather than by the evolution of their physical properties.

The extremely low dust fraction of iron measured at $[\mathrm{Fe} / \mathrm{H}] \lessgtr-2$ dex suggests that dust formation is very inefficient during the earliest stages of galactic chemical evolution. We considered the possibility that the main mechanisms of dust formation in galaxies may have a metallicity-dependent efficiency. Indeed, previous work already indicates that the dust formation efficiency in the ejecta of SNe II and in the winds of late-type giants may increase with metallicity. We argue that, in addition, the injection of iron-rich dust by type SNe Ia may yield a rise of $f_{\mathrm{Fe}}$ in the course of evolution. The metallicitydependent dust formation in SN II seems to be the most promising candidate for explaining the rise of $f_{\mathrm{Fe}}$ at $[\mathrm{Fe} / \mathrm{H}] \lessgtr-1 \mathrm{dex}$.

To reproduce the observed evolution of the dust content of DLA systems a simultaneous treatment of the main processes of dust formation, accretion and destruction is required. The observed increase of $f_{\mathrm{Fe}}$ with metallicity is contrary to the expectations of current chemical evolution models, which postulate or infer an approximately constant dust-to-metal ratio, with the exception of the simple, analytical models by Inoue (2003), which predict an increase of the dust-to-metal ratio $\mathcal{D} / \mathcal{Z}$, starting from very low values. The present results may represent a unique guideline for incorporating the dust component in well-tested models, particularly for the early stages of evolution. The successful implementation of the observed trends between $f_{\mathrm{Fe}}$, metallicity and cosmic time in realistic models will provide fresh clues on the formation redshift and nature of DLA galaxies.

Acknowledgements. I wish to thank Andrea Ferrara, Akio Inoue and Patrick Petitjean for their useful comments on the original version of the manuscript.

\section{References}

Anders, E., \& Grevesse, N. 1989, Geochim. Cosmochim. Acta, 53, 197

Bergeson, S. D., \& Lawler, J. E. 1993, ApJ, 408, 382

Boissé, P., Le Brun, V., Bergeron, J., \& Deharveng, J. M. 1998, A\&A, 333,841

Calura, F., Matteucci, F., \& Vladilo, G. 2003, MNRAS, 340, 59

Carilli, C. L., Lane, W., de Bruyn, A. G., Braun, R., \& Miley, G .K. 1996, AJ, 111, 1830

Carilli, C. L., Bertoldi, F., Menten, K. M., et al. 2000, ApJ, 533, L13

Centurión, M., Molaro, P., Vladilo, G., et al. 2003, A\&A, 403, 55

Chengalur, J. N., \& Kanekar, N. 2000, MNRAS, 318, 303

Clayton, D. D., Arnett, D., Kane, J., \& Meyer, B. S. 1997, ApJ, 486, 824

de la Varga, A., Reimers, D., Tytler, D., Barlow, T., \& Burles, S. 2000, A\&A, 363, 69

Dessauges-Zavadsky, M., Péroux, C., Kim, T.-S., D’Odorico, S., \& McMahon, R. G. 2003, MNRAS, 345, 447

Dunne, L., Eales, S., Ivison, R., Morgan, H., \& Edmunds, M. 2003, Nature, 424, 285

Dwek, E. 1998, ApJ, 501, 643

Dwek, E., \& Scalo, J. M. 1980, ApJ, 239, 193

Edmunds, M. G. 2001, MNRAS, 328, 223

Ellison, S. L., \& Lopez, S. 2001, A\&A, 380, 117

Elmhamdi, A., Danziger, I. J., Chugai, N., et al. 2003, MNRAS, 338, 939

Fan, X., Strauss, M. A., Schneider, D. P., et al. 2003, AJ, 25, 1649

Ferrarotti, A. S., \& Gail, H.-P. 2003, in Astrophysics of Dust, Estes Park, Colorado, May 26-30, ed. A. N. Witt

Franco, J., \& Cox, D. P. 1986, PASP, 98, 1076

Galliano, F., Madden, S. C., Jones, A. P., et al. 2003, A\&A, 407, 159

Ge, J., \& Bechtold, J. 1997, ApJ, 477, L73

Gratton, R., Carretta, E., Claudi, R., Lucatello, S., \& Barbieri, M. 2003, A\&A, 404, 187

Hirashita, H. 1999, ApJ, 510, L99

Hirashita, H., \& Ferrara, A. 2002, MNRAS, 337, 921 
Hou, J. L., Boissier, S., \& Prantzos, N. 2001, A\&A, 370, 23

Inoue, A. K. 2003, PASJ, 55, 901

Issa, M. R., MacLaren, I., \& Wolfendale, A. W. 1990, A\&A, 236, 237

Kanekar, N., \& Chengalur, J. N. 2003, A\&A, 399, 857

Kobayashi, C., Tsujimoto, T., Nomoto, K., Hachisu, I., \& Kato, M. 1998, ApJ, 503, L155

Lattimer, J. M., Schramm, D. N., \& Grossman, L. 1978, ApJ, 219, 230

Le Brun, F., Bergeron, J., Boissé, P., \& Deharveng, J. M. 1997, A\&A, 321, 733

Ledoux, C., Petitjean, P., \& Srianand, R. 2003, MNRAS, 346, 209

Ledoux, C., Srianand, R., \& Petitjean, P. 2002, A\&A, 392, 781

Levshakov, S. A., Molaro, P., Centurión, M., et al. 2000, A\&A, 361, 803

Lisenfeld, U., \& Ferrara, A. 1998, ApJ, 496, 145

Liszt, H. 2002, A\&A, 389, 393

Lopez, S., Reimers, D., D’Odorico, S., \& Prochaska, J. X. 2002, A\&A, 385,778

Lopez, S., Reimers, D., Rauch, M., Sargent, W. L. W., \& Smette, A. 1999, ApJ, 513, 598

Lu, L., Sargent, W. L. W., Barlow, T. A., Churchill, C. W., \& Vogt, S. 1996, ApJS, 107, 475

Meyer, D. M., Lanzetta, K. M., \& Wolfe, A. M. 1995, ApJ, 451, L13

Meyer, D. M., \& York, D. G. 1992, ApJ, 339, L121

Mishenina, T. V., Kovtyukh, V. V., Soubiran, C., Travaglio, C., \& Busso, M. 2002, A\&A, 396, 189

Molaro, P., Bonifacio, P., Centurión, M., et al. 2000, ApJ, 541, 54

Morgan, H. L., \& Edmunds, M. G. 2003, MNRAS, 343, 427

Moseley, S. H., Dwek, E., Glaccum, W., Graham, J. R., \& Loewenstein, R. F. 1989, Nature, 340, 697

Nissen, P. E., Chen, Y. Q., Asplund, M., \& Pettini, M. 2004, A\&A, 415, 993

Ossenkopf, V., Henning, Th., \& Mathis, J. S. 1992, A\&A, 261, 567

Pei, Y. C., Fall, S. M., \& Bechtold, J. 1991, ApJ, 378, 6

Péroux, C., Petitjean, P., Aracil, B., \& Srianand, R. 2002, New Astron., 7, 577

Petitjean, P., Srianand, R., \& Ledoux, C. 2000, A\&A, 364, L26

Pettini, M., Ellison, S. L., Steidel, C. C., \& Bowen, D. V. 1999, ApJ, 510,576
Pettini, M., Ellison, S. L., Steidel, C. C., Shapley, A. E., \& Bowen, D. V. 2000, ApJ, 532, 65

Pettini, M., Smith, L. J., Hunstead, R. W., \& King, D. L. 1994, ApJ, 426, 79

Primas, F., Brugamyer, E., Sneden, C., et al. 2000, The First Stars, ed. A. Weiss, T. G. Abel, \& V. Hill (Berlin: Springer), 51

Prochaska, J. X., Gawiser, E., Wolfe, A. M., Cooke, J., \& Gelino, D. 2003, ApJS, 147, 227

Prochaska, J. X., Henry, R. B. C., O’Meara, J. M., et al. 2002, PASP, 114,933

Prochaska, J. X., \& Wolfe, A. M. 1996, ApJ, 469, 403

Prochaska, J. X., \& Wolfe, A. M. 1997, ApJ, 474, 140

Prochaska, J. X., \& Wolfe, A. M. 1999, ApJS, 121, 369

Prochaska, J. X., \& Wolfe, A. M. 2002, ApJ, 566, 68

Prochaska, J. X., Wolfe, A. M., Tytler, D., et al. 2001, ApJS, 137, 21

Roth, K. C., \& Blades, J. C. 1995, ApJ, 445, L95

Savage, B. D., \& Sembach, K. R. 1996, ARA\&A, 34, 279

Sedlmayr, E. 1989, in Interstellar Dust, ed. L. J. Allamandola, \& A. G. G. M. Tielens (Dordrecht: Kluwer), IAU Symp., 135, 467

Schmidt, K.-H., \& Boller, T. 1993, Astron. Nachr., 314, 361

Silva, L. Granato, G. L., Bressan, A., \& Danese, L. 1998, ApJ, 509, 103

Sneden, C., Gratton, R., \& Crocker, D. A. 1991, A\&A, 246, 354

Spergel, D. N., Verde, L., Peiris, H .V., et al. 2003, ApJS, 148, 175

Todini, P., \& Ferrara, A. 2001, MNRAS, 325, 726

Turnshek, D. A., Rao, S., Nestor, D., et al. 2001, ApJ, 553, 288

Umeda, H., \& Nomoto, K. 2002, ApJ, 565, 385

Vladilo, G. 1998, ApJ, 493, 583 (Paper I)

Vladilo, G. 2002a, ApJ, 569, 295 (Paper II)

Vladilo, G. 2002b, A\&A, 391, 407 (Paper III)

Vladilo, G., Centurión, M., Bonifacio, P., \& Howk, J. C. 2001, ApJ, 557, 1007

Welty, D. E., Hobbs, L. M., Lauroesch, J. T., et al. 1999, ApJS, 124, 465

Wolfe, A. M., Turnshek, D. A., Smith, H. E., \& Cohen, R. D. 1986, ApJS, 61, 249

Wolfe, A. M., Prochaska, J. X., \& Gawiser, E. 2003a, ApJ, 593, 215

Wolfe, A. M., Gawiser, E., \& Prochaska, J. X. 2003b, ApJ, 593, 235 